\title{
Riesgos de escasez de agua en la ciudad de Huancayo al año 2030
}

\author{
Risk of water scarcity in Huancayo city on 2030
}

\section{RESUMEN}

Objetivos: Estimar los riesgos de escasez de agua en la ciudad de Huancayo (distritos de Huancayo, El Tambo y Chilca) al año 2030, mediante la recopilación y análisis de datos de oferta y demanda de agua. Métodos: Se desarrolló una investigación de alcance descriptivo, diseño transversal. Se utilizó el método de análisis del peligro de escasez de agua, considerando la oferta de agua para abastecimiento urbano, demanda doméstica y su balance, para la vulnerabilidad fue observada la conducta de uso y preparación frente a la escasez mediante la aplicación de una encuesta a jefes de familia de 1172 viviendas de la zona urbana de los distritos de Huancayo, El Tambo y Chilca; luego sistematizamos la información generando una base de datos a través del software ArcGis que tuvo como unidad de análisis espacial los sectores urbanos; para después estimar los riesgos expresados en sector y población afectada para un escenario actual y para el año 2030. Resultados: Las tendencias de crecimiento poblacional indican que la ciudad de Huancayo tendrá 429100 habitantes en el año 2030 , por lo tanto demandarán un volumen de 25,8 millones de metros cúbicos, superando a la oferta en $45 \%$ debido a pérdidas en la distribución, afectando de este modo a un $33,7 \%$ de la ciudad, que pertenecen a sectores de alto riesgo y donde se encuentran 42 centros educativos, 7 centros de salud y 12 centros comerciales. Conclusiones: Al año 2030 la población afectada por la escasez de agua ascenderá a 152432 habitantes, debido a un racionamiento de agua promedio de 2 horas menos a las actuales.

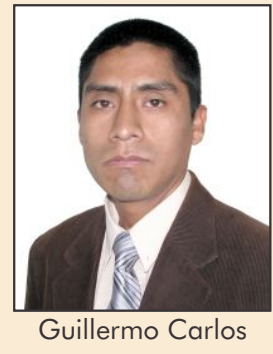

Palabras clave: Riesgo, escasez, agua, vulnerabilidad, consumo doméstico. 


\section{ABSTRACT}

Objectives: To estimate the risks of water scarcity in Huancayo city (Huancayo, El Tambo and Chilca districts) on 2030, by compilation and data analysis of water supply and demand. Methods: We developed a descriptive research, crosssectional design, we used the analysis method of water scarcity hazard, considering the water supply for the urban supplying, domestic demand and its balance, for the vulnerability was contemplated the use behavior and preparation addressing scarcity based on the application of a survey to householders of 1172 homes in the urban area of Huancayo, El Tambo and Chilca districts, then we systematized the information generating a database using ArcGis software, this had like spatial analysis unit the urban sectors, then estimating risks expressed in sector and in affected population to a current scenario for the 2030. Results: The population growth trends indicate that Huancayo city have 429100 population in 2030 and would demand a volume of 25,8 million cubic meters, outstripping the supply in $45 \%$, due to distribution losses, affecting in this way at the $33,7 \%$ of the city, belonging to high-risk sectors where there are 42 schools, 7 health centers and 12 commercial centers. Conclusions: In the year 2030 the affected population due to water scarcity will increase to 152432 people, due to water rationing average of 2 hours less to the current ones.

Keywords: Risks, scarcity, water, vulnerability, domestic consumption.

\section{INTRODUCCIÓN}

La escasez de agua puede ser una construcción social o consecuencia de la variación en los patrones de la oferta, derivados, por ejemplo, del cambio climático(1). En términos generales la escasez se debe a la combinación de muchos factores, los cuales están vinculados a aspectos como: la disponibilidad, distribución y su forma de consumo (2).

A nivel mundial se viene presentando casos de escasez de agua, estudios realizados revelan que en los próximos años países como; México tenderán hacia la pérdida de la seguridad hídrica (3). En India ya se presenta escasez física de agua (4). Así mismo, las proyecciones de disponibilidad de agua considerando el impacto del cambio climático estiman que un $70 \%$ de la población de América del Sur habitará en zonas con escasa oferta de agua en el año 2025 (5).

El Consejo Nacional del Ambiente el año 2003 indicó que la superficie total de glaciares en el Perú se redujo en un $22 \%$ en los últimos 20 años. Como consecuencia de esta merma, hemos perdido alrededor del $12 \%$ en volumen de agua. Se estima que para los años 2015 o 2020, todos los glaciares debajo de los 5 mil metros van a desaparecer derretidos (6). Ello afectará directamente nuestra principal fuente de agua, el río Shullcas; este deriva del nevado Huaytapallana, el acceso fácil y barato al agua estaría por terminar, significando una amenaza mayor que la pérdida de cualquier otro recurso natural (7).

La producción de agua potable resulta insuficiente para cubrir la creciente demanda poblacional, por ello se viene incrementado la presión a las fuentes subterráneas sin considerar que los acuíferos son una fuente no renovable a corto plazo y que su uso excesivo conduce inexorablemente a situaciones de mayor riesgo (8). Los elevados niveles de pérdidas de agua presentados por antigüedad del sistema, la limitada automatización de instalaciones, el inadecuado sistema de facturación y los desperdicios, hace que exista un déficit reflejado en racionamientos continuos en los últimos 10 años.

La escasez de agua afecta a la población de la zona urbana de los distritos de Huancayo, El Tambo y Chilca, tendiente a agravarse en 
los próximos años, siendo importante determinar en términos de riesgo; la implicancia de este peligro considerando condiciones sociales como: grado de preparación y respuesta para afrontarla. En tal sentido es necesario estimar los riesgos de escasez de agua en la ciudad de Huancayo con una proyección al año 2030, a fin de conocer la situación actual del recurso y su comportamiento en el futuro.

\section{MATERIAL Y MÉTODOS}

La investigación fue de alcance descriptivo, diseño transversal; utilizó el método de análisis del peligro de escasez de agua, considerando la oferta de agua para abastecimiento urbano, demanda doméstica y su balance; para la vulnerabilidad se contempló la conducta de uso y preparación frente a la escasez en base a la aplicación de una encuesta a jefes de familia de 1172 viviendas de la zona urbana de los distritos de Huancayo, El Tambo y Chilca, abarcando una extensión de 4436 hectáreas, divididas según el Plan de desarrollo Urbano de la Municipalidad Provincial de Huancayo en 20 sectores como se observa en la Figura 01.

\section{Evaluación de la escasez de agua}

Para el caso, fueron recopilados los datos históricos de los volúmenes netos mensuales de aporte por las fuentes superficiales y subterráneas que abastecen a la ciudad de Huancayo, registrados por la empresa SEDAM HUANCAYO y la Superintendencia Nacional de Servicios de Saneamiento (SUNASS) en el periodo 2000 - 2011; así como de los caudales promedio mensuales del río Shullcas desde 1985 hasta el 2011 registrados por la Administración Local de Agua Mantaro.

Se proyectó la oferta de agua al año 2030 empleando el modelo Autorregresivo

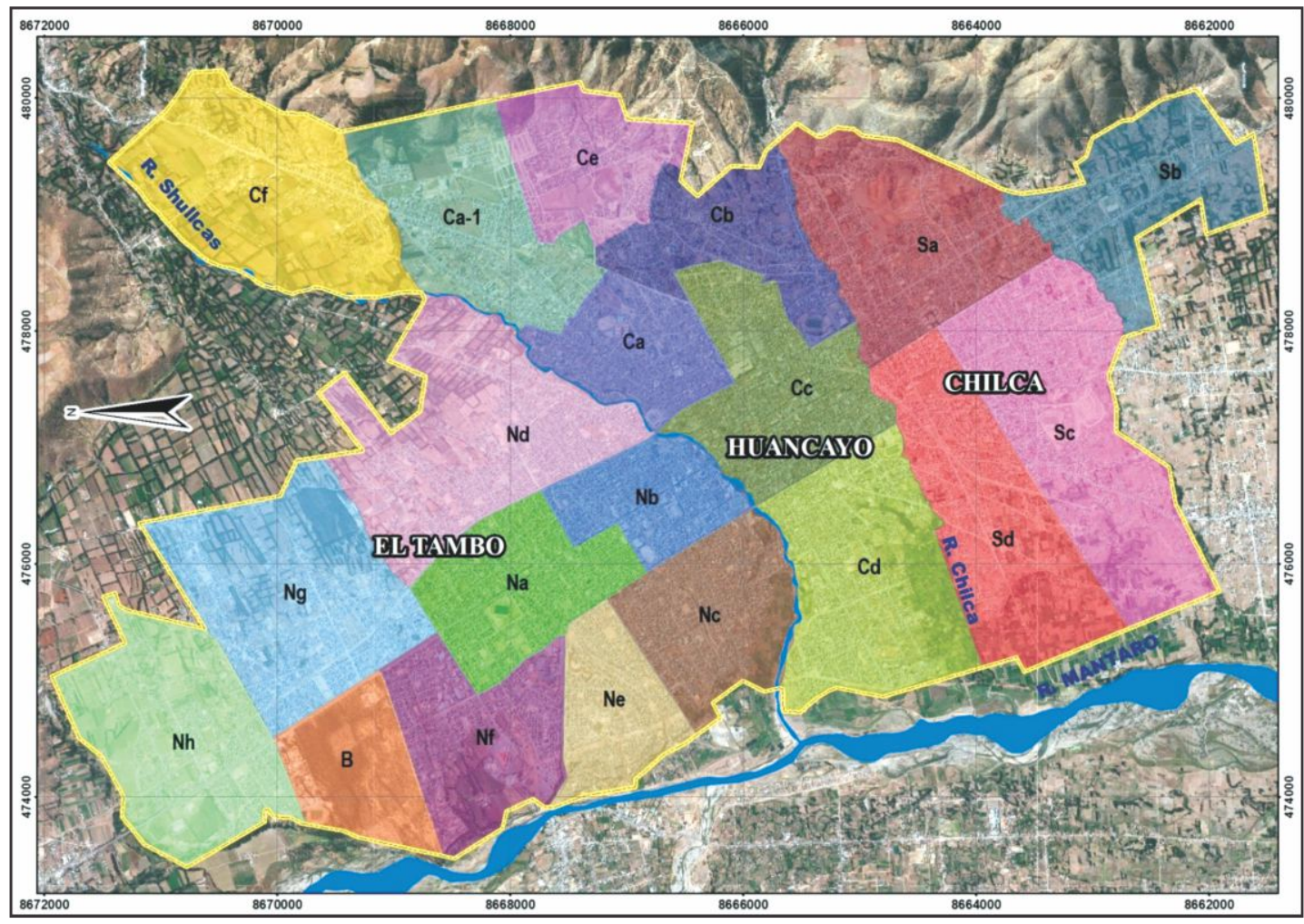

Figura 01: Sectores urbanos de los distritos de Huancayo, El Tambo y Chilca. 
Tabla 01: Funciones de crecimiento poblacional según distritos

\begin{tabular}{lll}
\hline \multicolumn{1}{c}{ Distrito } & \multicolumn{1}{c}{ Función } & $\mathrm{R}^{2}$ \\
\hline Huancayo & $y=-17,734 \times 2+2209,3 x+45958$ & 0,997 \\
El Tambo & $y=6,569 \times 2+2675,8 x+5213,4$ & 0,991 \\
Chilca & $y=-6,394 \times 2+1652,7 x+10414$ & 0,998 \\
\hline
\end{tabular}

Integrado de Media Móvil (ARIMA) mediante el uso del software SPSS, considerando la ecuación básica, $w_{t}=\nabla^{d} z_{t}$ donde $\nabla^{d}$ representa las diferencias de orden " $d$ " del proceso $\mathrm{Zt}(9)$.

También recopilamos datos de censos poblacionales realizados por el Instituto Nacional de Estadística e Informática (INEI) entre los años 1961 a 2011, evaluando el crecimiento poblacional mediante regresión cuadrática por distrito y proyectándolo al año 2030, representados para cada caso en la Tabla 01.

El consumo per cápita se calculó en base a un modelo propuesto, siendo: $C_{p}=C_{i}+\frac{1}{x} C_{f}$ Donde, "Cp" es el consumo per-cápita, "Ci" es el consumo individual y " $\mathrm{Cf}$ " es el consumo colectivo, " $x$ " representa el número de personas por vivienda. A su vez el consumo se debe a tres factores; el número de repeticiones por día (nx), tiempo de uso del agua (tx) y el volumen de agua por minuto según uso $(V x)$, así se tiene: $C_{x}=n_{x} \times t_{x} \times V_{x}$

Se identificó 7 usos domésticos de agua, bañarse, lavarse los dientes, lavar la ropa, lavar utensilios, regar plantas, lavar acera y lavar carro, para el cual se recopiló, el tiempo medio según uso mediante una encuesta a 1172 jefes de familia elegidas completamente al azar a un nivel de confiabilidad del 95\%, distribuidas según sectores urbanos.

El balance de agua fue categorizado en función al valor porcentual en el que la demanda supera a la oferta, obteniendo la situación de escasez de agua según sector urbano.

Tabla 02: Tipos de vulnerabilidad e indicadores

\begin{tabular}{cl}
\hline Tipo de vulnerabilidad & Indicador \\
\hline $\begin{array}{c}\text { Vulnerabilidad educativa miembro de la familia asistió a eventos de capacitación en manejo } \\
\text { (VE) }\end{array}$ & $\begin{array}{l}\text { Ne agua } \\
\text { Nivel de conocimiento de la problemática del nevado Huaytapallana }\end{array}$ \\
Vulnerabilidad física de & $\begin{array}{l}\text { Envases de almacenamiento de agua con que cuentan las viviendas. } \\
\text { abastecimiento }\end{array}$ \\
(VF) & $\begin{array}{l}\text { Estado de las instalaciones sanitarias (conexión y griferías) de las viviendas. } \\
\text { Horas continuas de agua con que cuenta su vivienda. }\end{array}$ \\
Vulnerabilidad cultural de presión de agua en viviendas. \\
(VC)
\end{tabular}




\section{Evaluación de la vulnerabilidad}

Se consideró el valor porcentual del caso que reduce la vulnerabilidad según tipos e indicadores presentados en la Tabla 02, recogidos por la encuesta antes mencionada, obteniendo el valor total como el promedio según tipo y para cada sector urbano.

\section{Estimación de riesgos}

Se empleó el software ArcGis para generar una base de datos espacial y la determinación de los sectores en riesgo, estimando el impacto social, utilizando la metodología del Instituto Nacional de Defensa Civil (INDECI), (10). En la presente se trabajó con los indicadores; población total afectada, racionamiento de agua e infraestructura social a afectarse (centros educativos, centros de salud, y centros comerciales), por ser impactos inmediatos.

\section{RESULTADOS}

\section{Peligro de escasez de agua}

La Figura 02, muestra la tendencia del

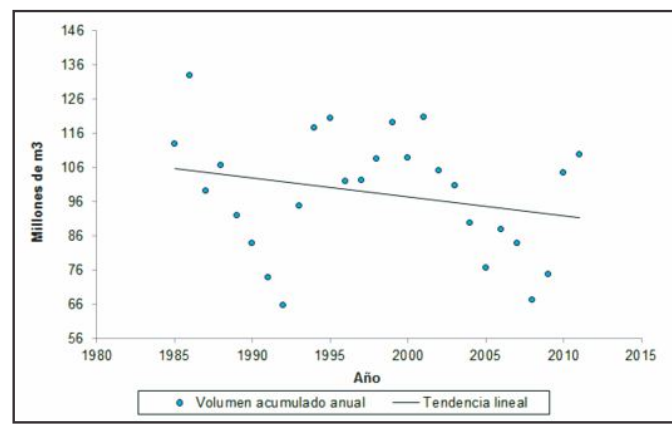

Figura 02: Tendencia del volumen acumulado anual

volumen anual de aporte del río Shullcas para el periodo 1985 a 2011, la cual es decreciente, significando una reducción promedio de -128 120,05 m3/año equivalente a -4 lt/s al año.

La Figura 03, muestra el aporte volumétrico por tipo de fuente de abastecimiento donde; las fuentes subterráneas actualmente aportan 45\%, superior al 35\% que contribuía, hasta antes del año 2004 y las fuentes superficiales actualmente han estabilizado su aporte en un $55 \%$.

La oferta de agua es afectada negativamente, debido al porcentaje de pérdidas cuyo valor promedio es de 45,8\%, que reduce el volumen captado de 30,9 a 14,2 millones de m3.

El Modelo ARIMA $(1,0,0) \times(0,1,1)$ fue el que

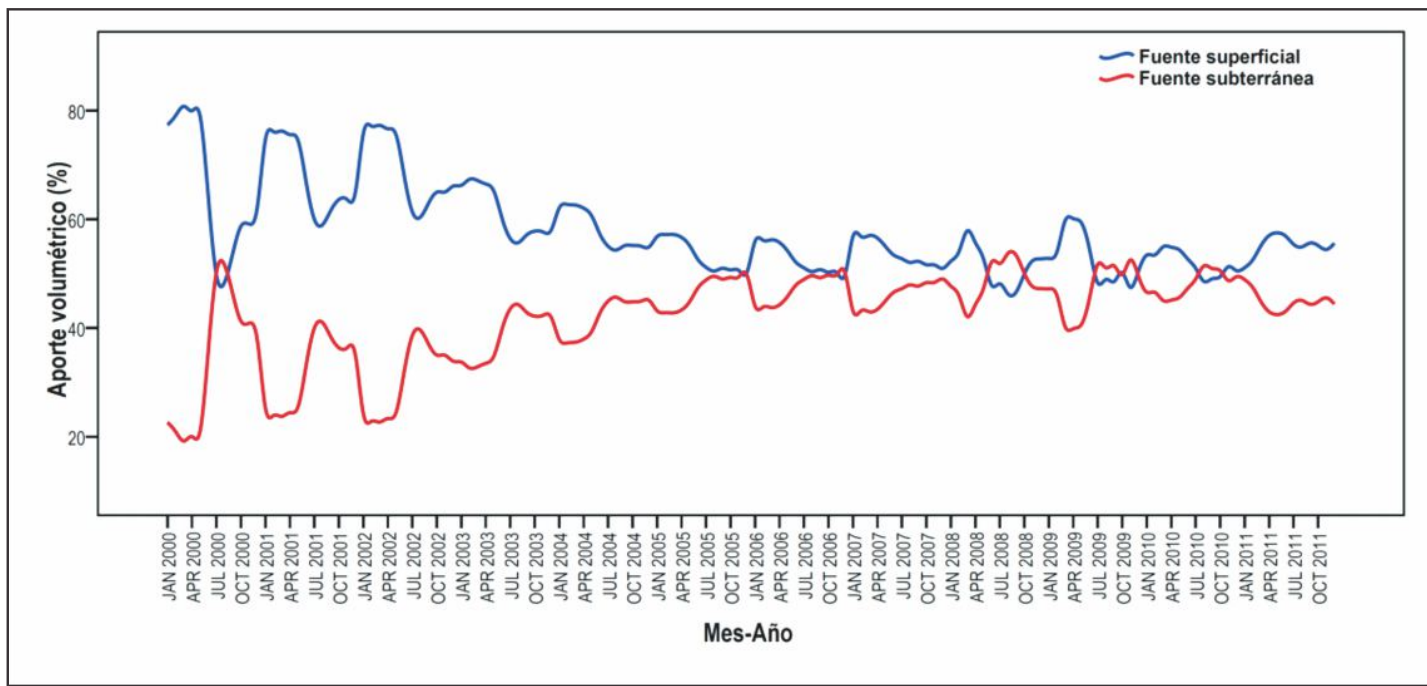

Figura 03: Aporte volumétrico porcentual según tipo de fuente (2000-2011) 


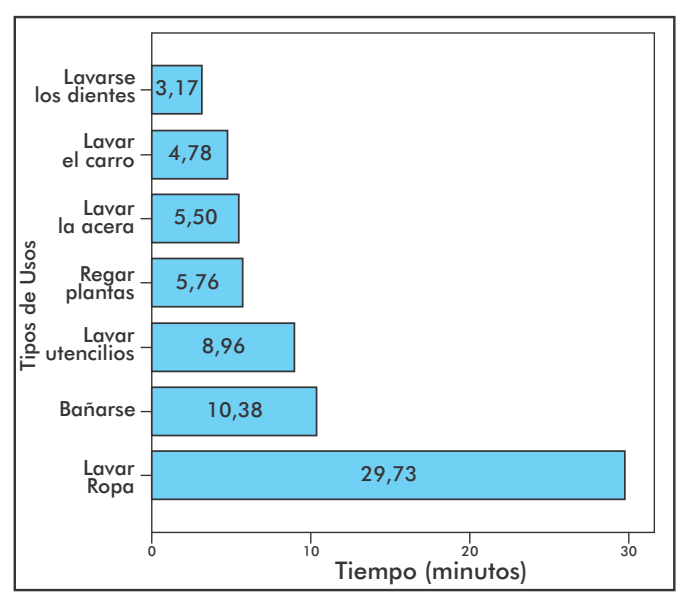

Figura 04: Tiempo promedio de gasto de agua según tipo uso

mejor representaba la serie por tener un $80,5 \%$ de grado de ajuste y estima un volumen anual acumulado para el año 2030 de 17,8 millones de m3.

El tiempo promedio según los usos identificados están registrados en la Figura 04, que se emplearon para determinar el consumo promedio de agua en la ciudad de Huancayo, siendo de 164,75 lt/hab/día y que varían según el sector urbano, como se puede ver en la Figura 05.

La Figura 06, muestra la comparación de oferta y demanda de agua, donde la ciudad de Huancayo pasaría de tener actualmente 344580 habitantes a contar con 429100 en el año 2030, demandando un volumen de 25,8 millones de m3 superando en $45 \%$ la oferta de agua para dicho año.

La Figura 07, muestra los sectores urbanos que presentan escasez y las áreas que se incorporarán a dicha condición, es así que el $21,5 \%$ del área de la ciudad de Huancayo pertenece a zonas de peligro muy alto, y al año 2030 , ascenderá a un $26,4 \%$.

\section{Evaluación de la vulnerabilidad}

Un 93,7\% de la ciudad de Huancayo pertenecen a zonas de vulnerabilidad media y solo un $2,3 \%$ están en vulnerabilidad baja,

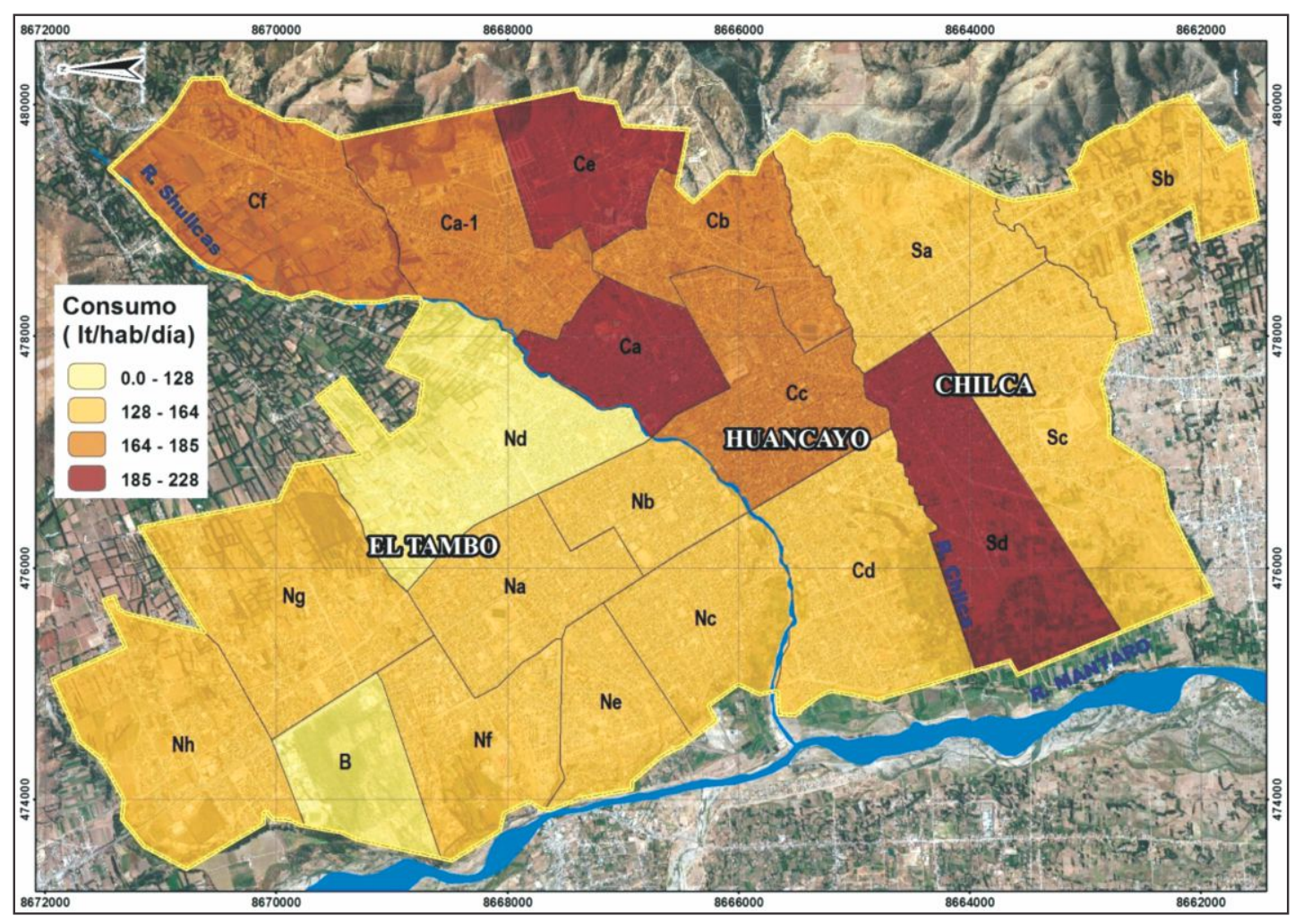

Figura 05: Sectores urbanos según consumo doméstico de agua per-cápita. 


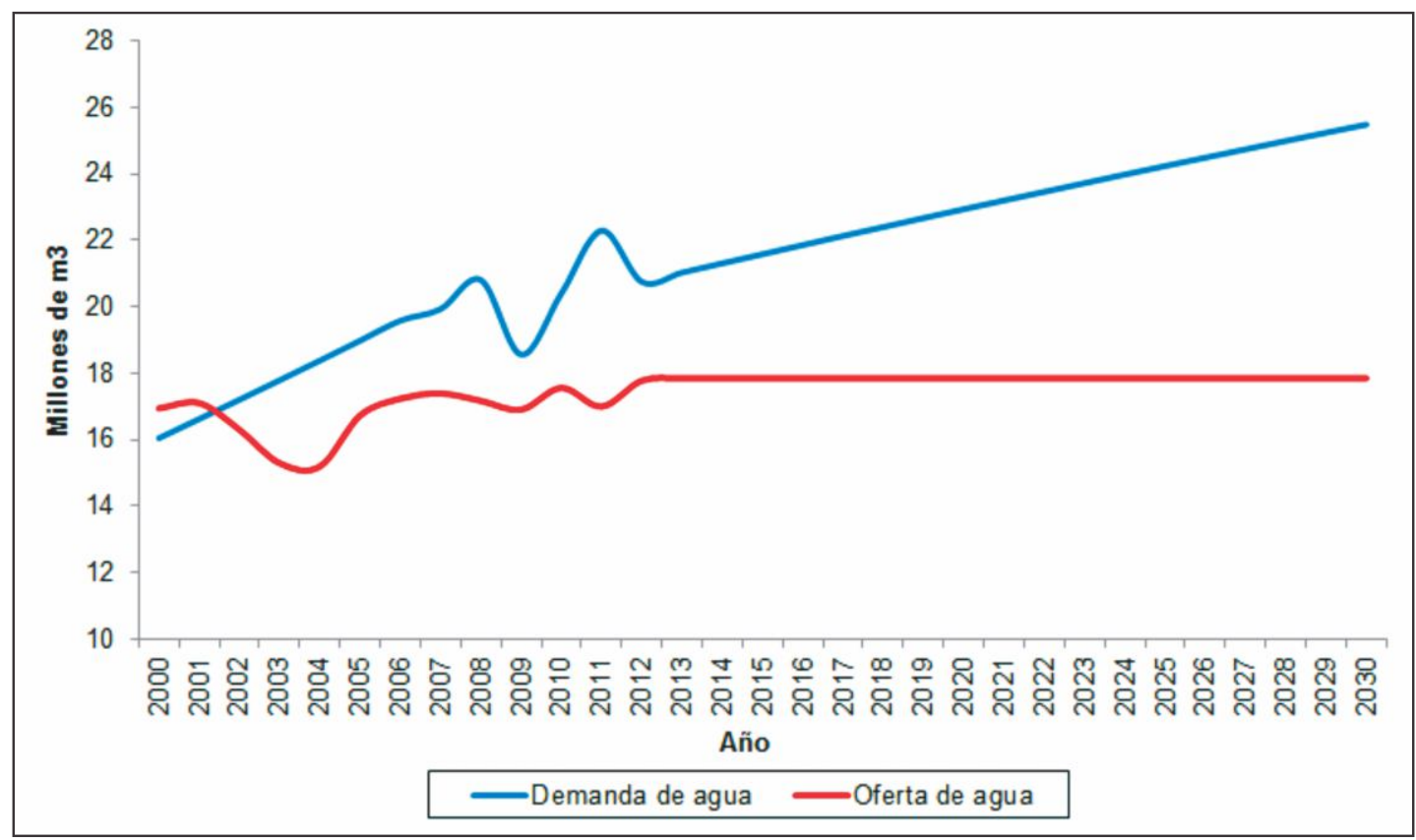

Figura 06: Comparación de la oferta y demanda de agua.

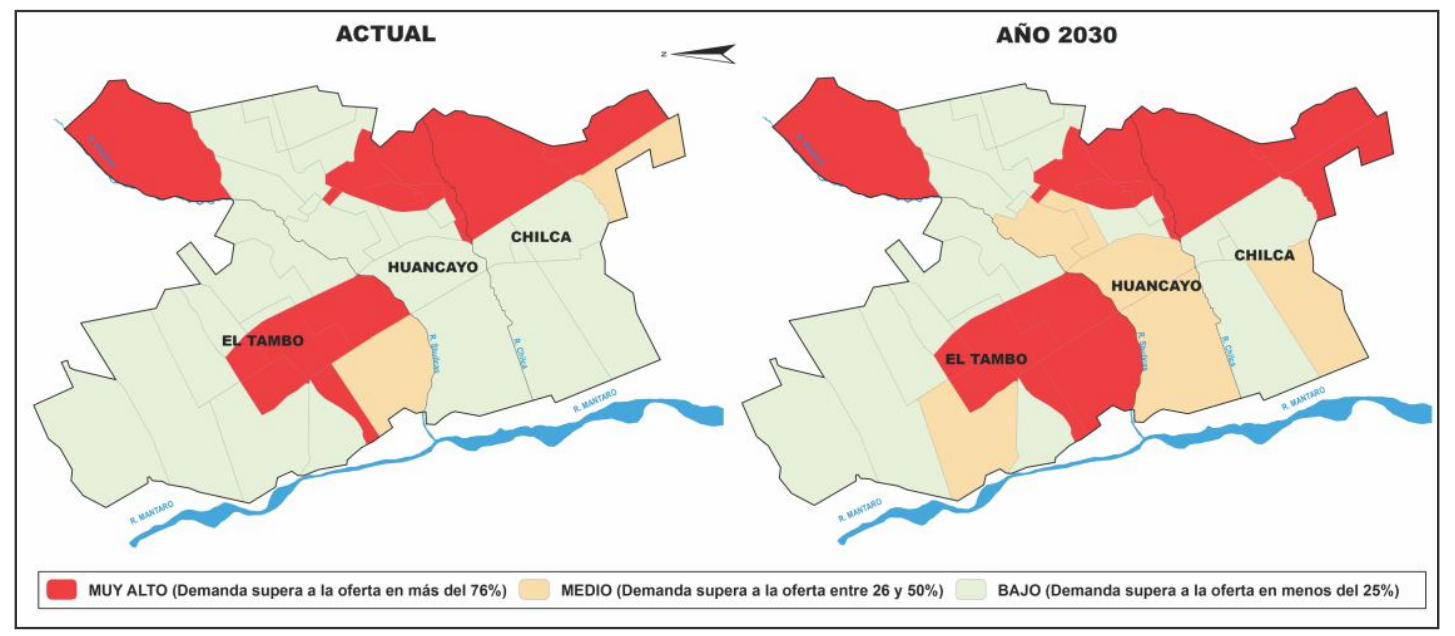

Figura 07: Peligro de escasez de agua para los años 2011 y 2030.

de estos; la vulnerabilidad educativa y cultural son las predominantes en el distrito de Huancayo (Figura 08) y la vulnerabilidad educativa y económica representa un nivel alto en los distritos de El Tambo y Chilca, tal como se muestra en la Figura 09 y 10.

\section{Estimación de riesgos}

La Figura 11, muestra que en la actualidad existe un $27,5 \%$ del área de estudio en zona de riesgo alto, $6,2 \%$ en riesgo medio y $66,3 \%$ en riesgo bajo, los cuales variarán al año 2030 presentándose; $33,7 \%$ en riesgo alto, $21,3 \%$ en riesgo medio y $45 \%$ en riesgo bajo.

La Figura 12, muestra la variación en la continuidad de agua, donde se reducirá según el sector, un promedio de 2 horas al año 2030, con respecto a la continuidad presentada actualmente. 


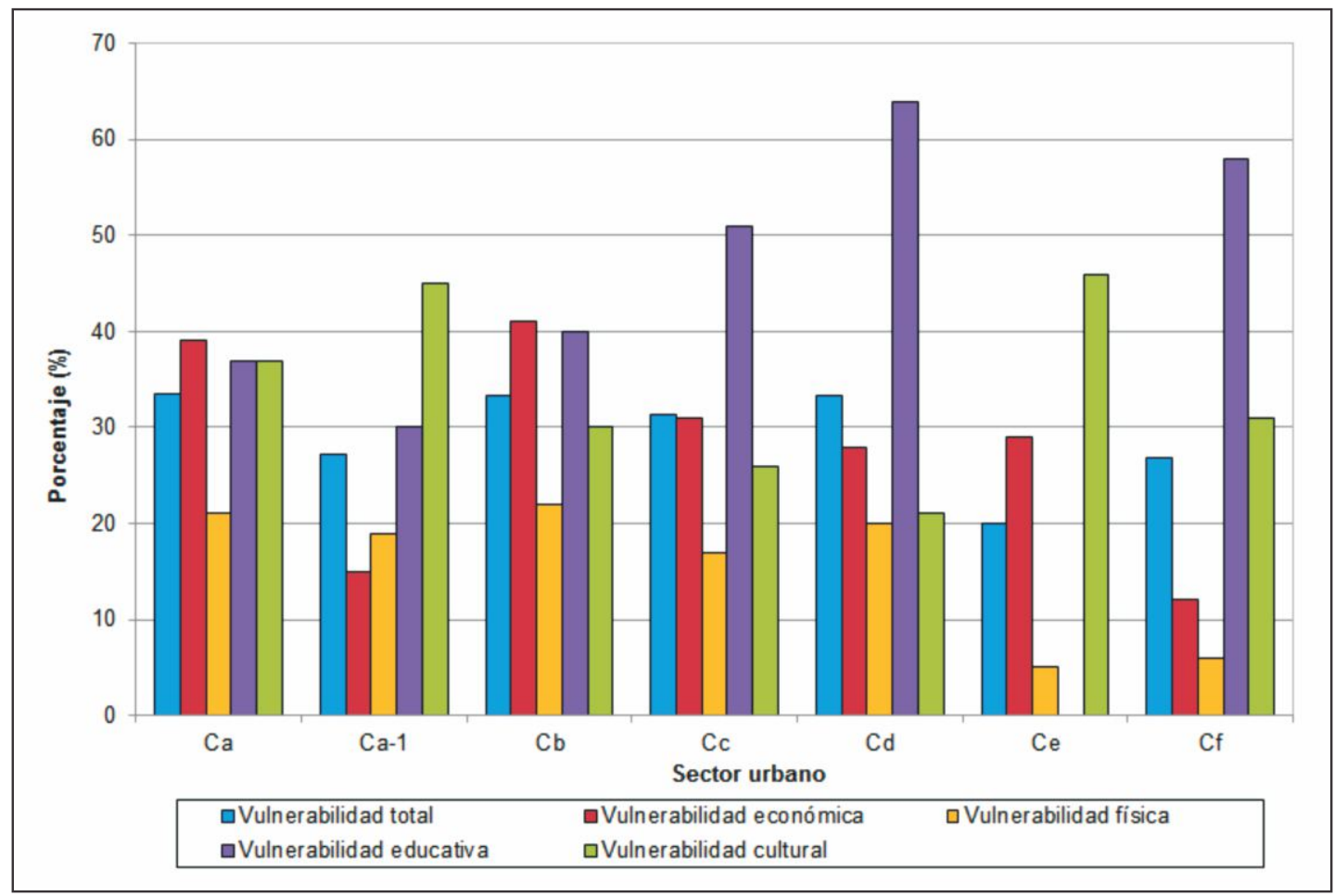

Figura 08: Vulnerabilidad a escasez de agua por sectores urbanos del distrito de Huancayo

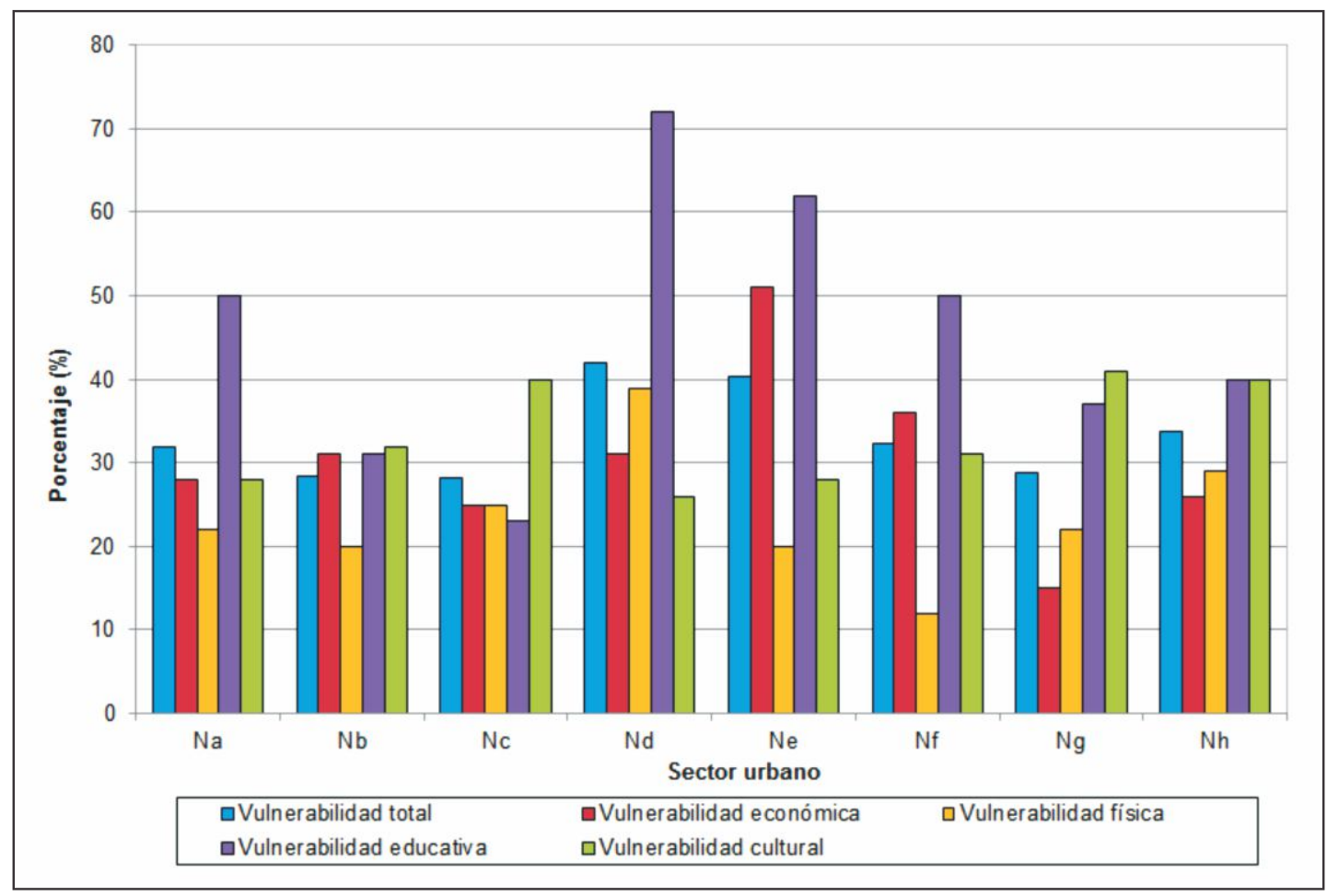

Figura 09: Vulnerabilidad a escasez de agua por sectores urbanos del distrito de El Tambo 


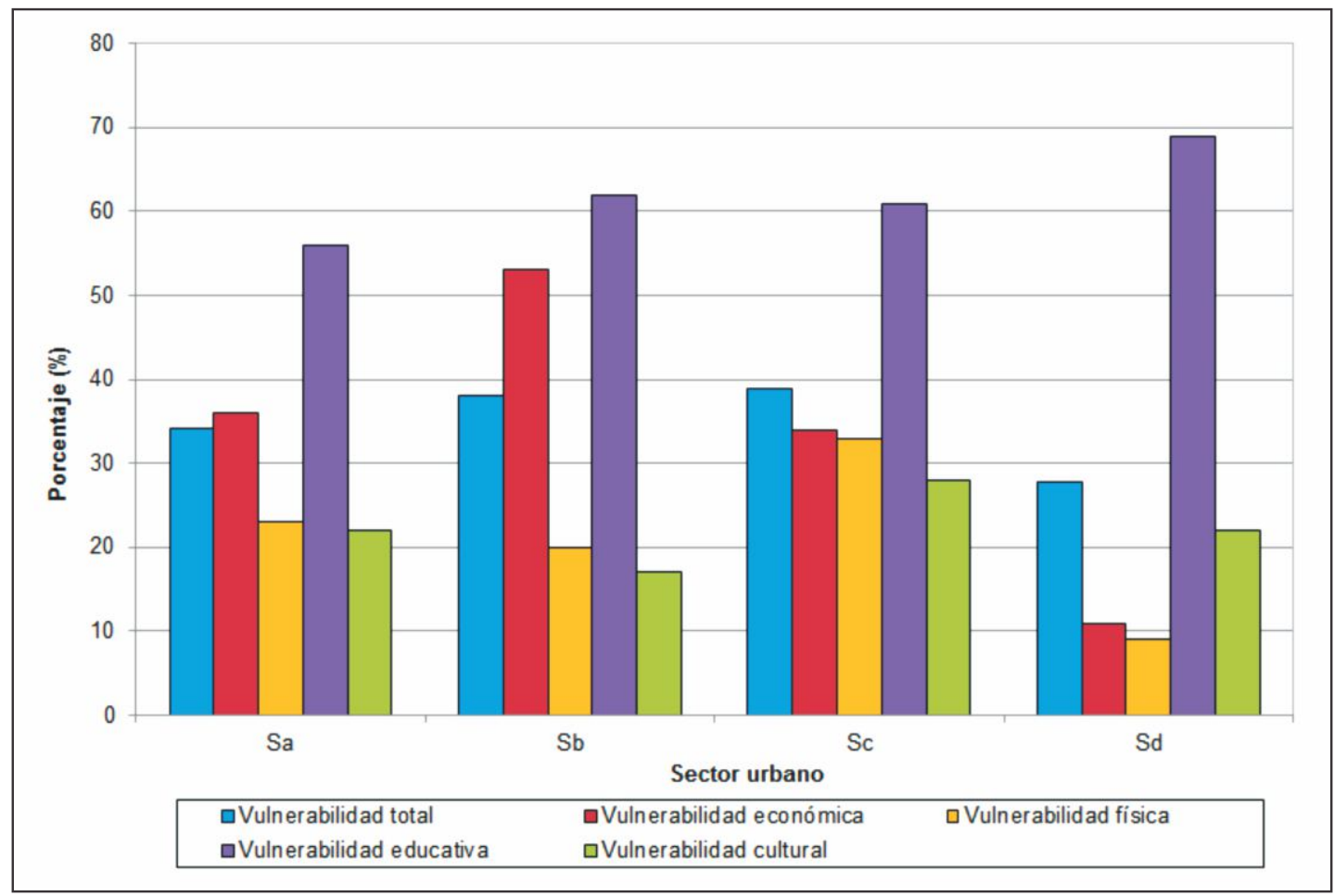

Figura 10: Vulnerabilidad a escasez de agua por sectores urbanos del distrito de Chilca.

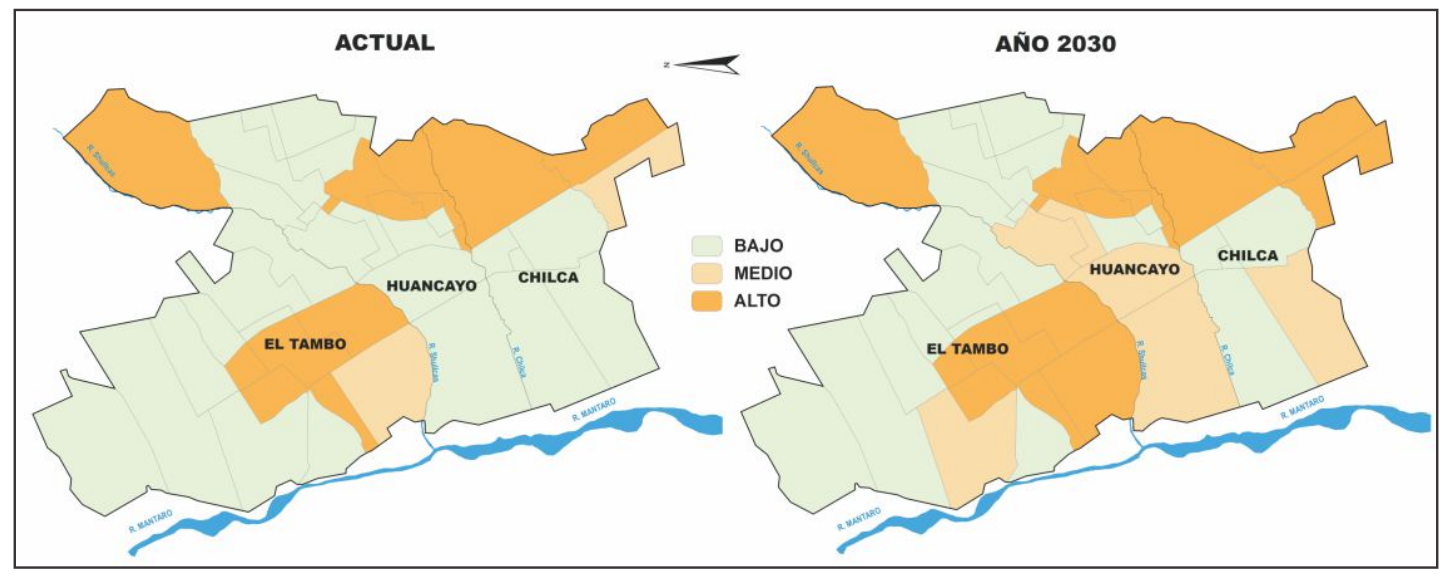

Figura 11: Zonas de riesgo de escasez de agua actual y proyectado al año 2030.

La Figura 13, muestra la infraestructura social ubicada dentro de las zonas de riesgo, de estas la infraestructura principal que se verá afectada al año 2030, serían; 42 centros educativos, 7 centros de salud y 12 de comercio.

\section{DISCUSIÓN}

El volumen de abastecimiento afectado por las pérdidas es superado por la demanda en un $31 \%$ actualmente y en el año 2030 será superado en $45 \%$. En el caso de no presentarse pérdidas en el sistema de distribución, hoy en día superaríamos la demanda en un $30 \%$ y al 2030 en $21,5 \%$, no 


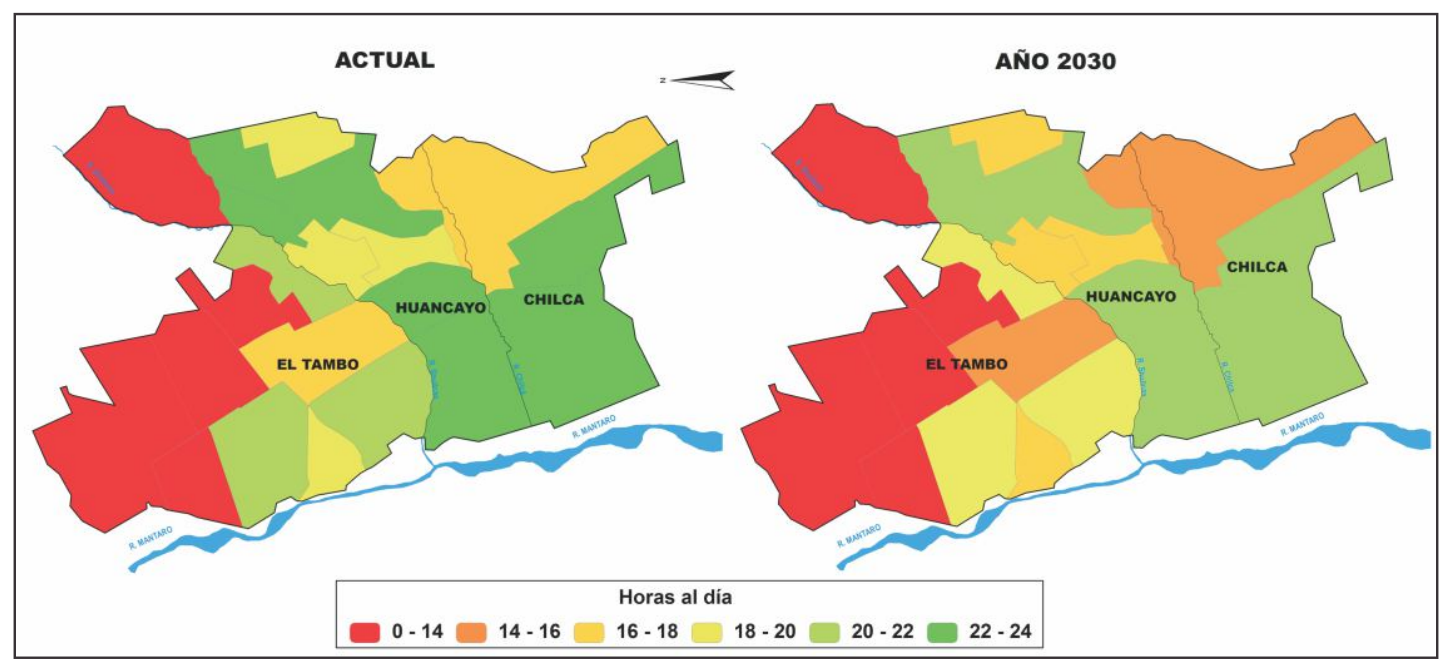

Figura 12: Variación de la continuidad de agua debido a la escasez

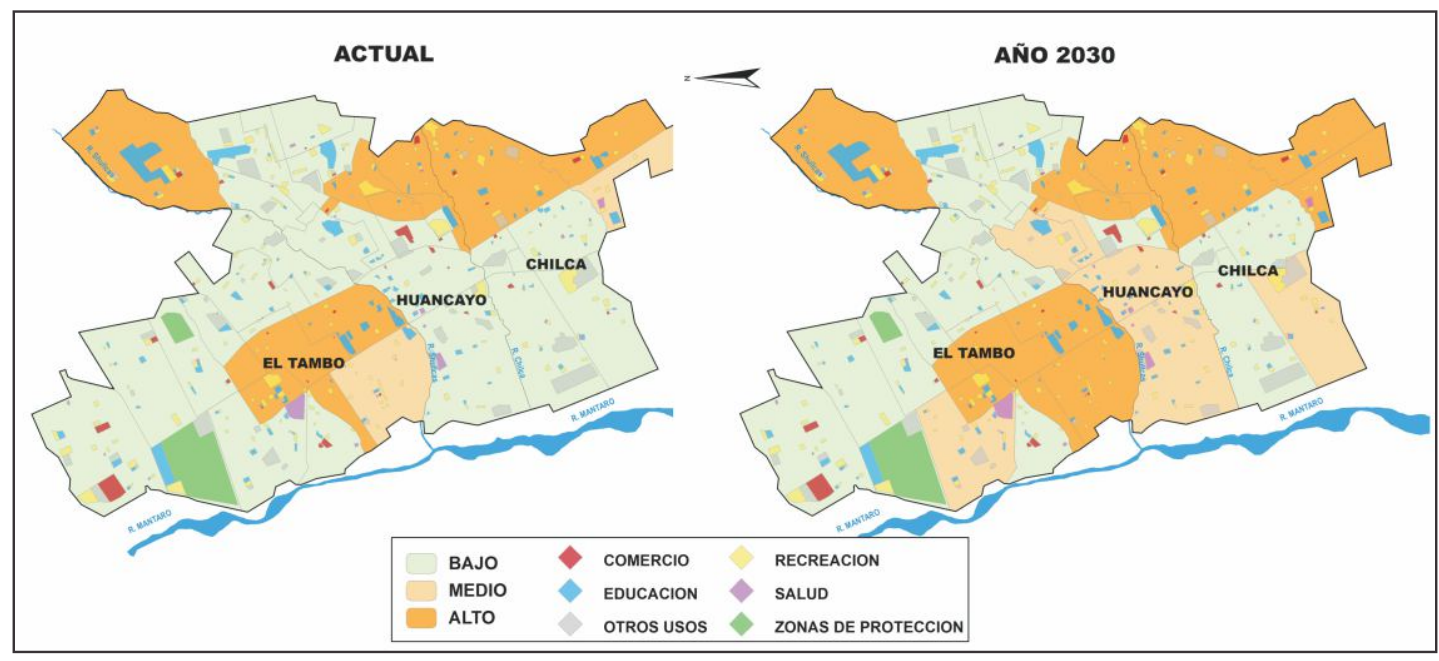

Figura 13: Infraestructura social afectada según zonas de riesgo.

presentándose riesgo alguno de escasez. Según Soto, señala que esta situación se ha generado por el crecimiento no planificado de la ciudad y la falta de mantenimiento del sistema, así como la falta del control de usos clandestinos (11).

El volumen disponible actual es menor al promedio de consumo $60 \mathrm{~m} 3 / \mathrm{hab} /$ año y en el año 2030 estará por debajo del consumo mínimo registrado en la actualidad equivalente a $43 \mathrm{~m} 3 / \mathrm{hab} / \mathrm{año}$.

El volumen autorizado captado del río Shullcas para fines de abastecimiento es de
$550 \mathrm{lt} / \mathrm{s}$, la oferta es complementada con la producción de pozos tubulares (aguas subterráneas). Esto obliga a elevar la presión año a año a las fuentes subterráneas para incrementar el volumen de oferta, siendo la salida más efectiva para enfrentar la escasez temporal de agua, pero que en el tiempo podría elevar el riesgo de escasez. Costa (12) al respecto, afirma que la excesiva presión sobre una fuente de agua puede conducir a su desaparición.

Según un estudio de variaciones de consumo en poblaciones del medio rural realizado el año 1987, en la zona de Palian 
(sector $\mathrm{Cf}$ ) se tenía una dotación per-cápita para consumo de agua de 68 lt/hab/día (13). En la actualidad el consumo es de 176,24 lt/hab/día, es decir se incrementó en 2,6 veces en 25 años, así mismo en ese mismo intervalo de tiempo la población de la ciudad de Huancayo incremento en 56\% su población. Se comprende bajo el caso citado que la población ha crecido pero ha incrementado mucho más su gasto, según Howard, et al (14) esto debió suceder por que la cobertura del servicio de agua potable pasó a estar dentro de casa reduciendo la valoración del agua e incrementando su gasto. Es así, que 6 sectores de la ciudad de Huancayo superan el promedio de consumo per-cápita nacional de 165 lt/hab/día (15).

Los sectores con peligro muy alto y medio abarcan principalmente las zonas céntricas de los tres distritos mientras que las zonas periféricas se catalogan en peligro bajo, esto se debe al alto consumo y alta densidad poblacional.

La vulnerabilidad educativa y económica, son las que tienen valores superiores al $50 \%$, esto implica que dichos sectores no están informados sobre la problemática local del agua y que a su vez consideran que pagan un alto costo por el agua, pese a que las empresas prestadoras públicas ofertan el agua más barata (16). La vulnerabilidad física y cultural presenta valores bajos, puesto que sus viviendas están preparadas para afrontar escasez y también realizan prácticas de ahorro y reutilización del agua.

En el año 2030 un 33,7\% de la ciudad de Huancayo se encontraría en zonas de riesgo alto, esto se debe, a que actualmente nos encontramos frente a una escasez económico-institucional de agua, que es el producto de la confluencia de limitado acceso al financiamiento, políticas de administración del recurso y al crecimiento poblacional, produciendo un incremento de consumo que serán complicadas de enfrentar bajo las experiencias de años pasados donde el problema se acrecentó por la reducción de agua en fuentes de abastecimiento (17).

\section{Agradecimientos}

A la Universidad Continental por su apoyo económico en el desarrollo de este trabajo de investigación; a Tito Mallma Capcha, Carlos Orihuela Villavicencio, Diana Córdova Torres, José Castañeda Cahuana, Miguel Angel Varillas Huaynalaya, Arturo Anco Arroyo, por brindar su apoyo técnico y a los estudiantes: Nohely Ventosilla, Ingrid Vilchez, Diego Casas, Frank Ramos, Gabriela Flores, Izamar Flores, Edson Vicuña, Ruben Manturano, Luisa Veliz, Sumaya Carrillo, Jorge Nishihara, y Víctor Ascue, por la colaboración en la recopilación de datos a través de la aplicación de un cuestionario en los distritos de Huancayo, El Tambo y Chilca.

\section{REFERENCIAS BIBLIOGRÁFICAS}

1. Organización de las Naciones Unidas para la Agricultura y la Alimentación (FAO). Coping with water scarcity challenge of the twenty-first century. Informe día Mundial del Agua.Roma: UN-Water; 2007.

2. Gaona T, et al. Planeación urbana y regional - Un enfoque hacia la sustentabilidad. México DF: Universidad Autónoma de Baja California; 2005.

3. Ávila P. Vulnerabilidad socioambiental, seguridad hídrica y escenarios de crisis por el agua en México. Ciencias. 2008; (90): 46-57.

4. De Les Valls JM. Estudio y análisis de la escasez física y económica de agua dulce en la India en función de la oferta y de la demanda [Tesis para optar el grado de Ingeniero de Organización Industrial]. Cataluña: Universidad Politécnica de Cataluña; 2011.

5. Smith MD. Sólo tenemos un planeta. $2 d a$ Ed. Lima: Intermediate Technology Publications Ltd; 2007. 
6. Ercilio F, Rodríguez S. Desafíos del Derecho Humano al Agua en el Perú. Centro de asesoría laboral del Perú. Lima: Centro de Asesoría Laboral del Perú; 2005.

7. Morrison J, Morikawa M, Murphy $M$, Schulte P. Water scarcity \& climate change: Growing Risks for Businesses \& Investors. Boston: Pacific Institute; 2009.

8. Velasco I, Montesillo JL. Elementos en la gestión de cuencas en condiciones de sequía. Gestión y Política Pública. 2007; 16(1): 5-27.

9. Chereque W. Hidrología para estudiantes de ingeniería civil. Lima: Pontificia Universidad Católica del Perú; 1989.

10. Bisbal A, Picón J, Casaverde M, Jauregui F, Achayhua R, Sanches R, Masana M. Manual básico para la estimación del riesgo. Lima: Instituto Nacional de Defensa Civil (INDECI); 2006.

11. Soto G. Agua: Tarifas, escasez y sustentabilidad en las megaciudades. lera Ed. México: Universidad Iberoamericana; 2007.

12. Costa C, Domínguez E, Gonzalo $H$, Vanegas R. El índice de escasez de agua ¿Un indicador de crisis o una alerta para orientar la gestión del recursos hídrico? Revista de Ingeniería. 2005; (22): $104-$ 111.

13. División de Saneamiento Básico Rural (DISABAR), Ministerio de Salud del Perú. Estudio de variaciones de consumo en poblaciones del medio rural. Lima: Centro Panamericano de Ingeniería Sanitaria y Ciencias del Ambiente; 1987.

14. Howard G., Bartram J. Domestic water quantify service level and health. Ginebra: WHO documentproductionservices; 2003.

15. Dirección Nacional de Urbanismo. Sistema Nacional de Estándares de Urbanismo Propuesta Preliminar Documento de trabajo [Internet]. Lima: Ministerio de Vivienda, Construcción y Saneamiento; 2011 [citado 09/02/12]. Disponible en: http://fenix.vivienda.gob. pe /OBSERVATORIO/Documentos /Normativa/NormasPropuestas/Estand aresUrbanismo/CAPITULOIII.pdf
16. Programa de las Naciones Unidas para el Desarrollo (PNUD). Informe sobre Desarrollo Humano 2006 - Escasez de agua, riesgo y vulnerabilidad. Nueva York: AGS CustomGraphics; 2006.

17. Mayo R. Abastecimiento de agua en Huancayo peligra por déficit en las lagunas. El Comercio. 2008 mayo 8. p.17.

Correo electrónico: carlos.gomez.miguel@gmail.com rgrijalva@continental.edu.pe 